\title{
LIGHTCONE $2013^{+}$: Editorial
}

\author{
Venturing Off the Lightcone: Local Versus Global Features
}

Received: 9 April 2014 / Accepted: 11 April 2014 / Published online: 27 April 2014

(C) Springer-Verlag Wien 2014

\section{LIGHTCONE $2013^{+}$: Summary}

The LIGHTCONE Conferences explore annually over a span of more than two decades several topics related to lightcone quantization methods to physical problems, ranging from pure quantum field theory to QCD, hadron models, and beyond.

The $\mathrm{LC}_{2013^{+}}$meeting of the International Light Cone Advisory Committee, Inc. was organized by the Institut für Theoretische Physik II, Ruhr-Universität Bochum, Germany, and was held at "Skiathos Palace" Hotel, in Skiathos, Greece, May 20-25, 2013. The organizing committee consisted of N. G. Stefanis (chair), E. Epelbaum, H. Krebs, M. Polyakov, and A. I. Karanikas (Athens, Greece). The following topics were covered: (i) Wilson lines off-the-lightcone, (ii) lightcone field theory, (iii) AdS/CFT-theory and applications, (iv) hadron structure in QCD, (v) effective theories, and (vi) lattice simulations. The LC2013 workshop had a satellite meeting attached to it during the last one and a half days, which addressed issues around the use of Bethe-Salpeter equations and their relations to Euclidean- and Minkowski-based approaches. The scientific organization of this event was in the hands of B. L. G. Bakker, T. Frederico, and L. Tomio.

LIGHTCONE $2013^{+}$was planned as a purely theoretical meeting with the main aim to bring together researchers from around the world working on various issues pertaining to QCD and hadron physics. The purpose was to provoke thought and collaborative work encouraging the cross-talk between scientists that use different concepts and calculational techniques-from theory to phenomenology. LC2013 gathered in total 56 participants and avoided by concept parallel and poster sessions. Each day began with 2-3 thematic keynote talks of $40 \mathrm{~min}$, followed by $30 \mathrm{~min}$ talks. The time schedule was arranged in such a way as to give enough time for informal discussions in the breaks. The main subjects of the Workshop were reviewed in three Special Lectures with a duration of $45 \mathrm{~min}$ each. The three lecturers were S. J. Brodsky (Hadron Physics on the Lightfront), A. V. Efremov (TMD Phenomenology), and D. Müller (GPD Phenomenology). These lectures were surrounded by other keynote talks covering various areas of current interest ranging from the use of Wilson lines in TMD formulations pertaining to semi-inclusive DIS and Drel-Yan processes, etc., to DVCS phenomenology, jet quenching, Chiral Perturbation Theory, lightfront QCD, and AdS/CFT/QCD theory and its applications to hadrons.

This special issue of Few Body Systems contains the contributions of the participants, each relating to the themes of the conference in a unique way, providing insights into both, problems and solutions. Below, it is not intended to provide a comprehensive summary of the presented material, but to highlight some key issues.

Several aspects of TMD theory and selected phenomenological applications were discussed by B. Pasquini, while P. Mulders concentrated on theoretical issues related to the factorization of TMD quantities and their

N. G. Stefanis $(\varangle)$

Institut für Theoretische Physik II, Ruhr-Universität Bochum, 44780 Bochum, Germany

E-mail: stefanis@tp2.ruhr-uni-bochum.de

Tel.: +49-234-3223724

Fax: +49-234-3214967 
path dependence in terms of Wilson lines extending off the lightcone. Within the same context, A. Vladimirov presented the derivation of the soft factor, involved in the TMD definition to render it free from rapidity divergences, using techniques akin to those of SCET. An interesting talk on intense laser fields using lightfront quantization to QED was given by A. Ilderton, while the dynamical radiation reaction in lightfront QED was studied by Torgrimsson. X. Zhao presented a nonperturbative first-principles approach to time-dependent problems in quantum field theory and applied it to the study of electron acceleration and the associated photon emission in QED.

Lightfront techniques were also employed by H.-M. Choi and C.-R. Ji to analyze the twist-two and twistthree quark-antiquark distribution amplitudes with even chirality and compute the vector-meson decay constant. The calculation of the twist-two distribution amplitude for the longitudinally polarized $\rho$ meson was also considered by A. V. Pimikov using the framework of QCD sum rules with nonlocal condensates. H. Moutarde and collaborators performed detailed analysis of DVCS in the near forward kinematic region with the aim to assess GPDs. They found that $\mathcal{O}\left(\alpha_{s}\right)$ corrections and gluonic contributions are not negligible, even in the energy range accessible at JLab12 and at the COMPASS-II experiment at CERN. B. L. G. Bakker and C.-R. Ji proposed DVCS as a tool of studying the hadron structure in exclusive processes. Using a quark-diquark model of a proton, they discussed the analytic parametrization of the Compton tensor amplitude in terms of independent Compton form factors. Numerical calculations could reveal the relative importance of these form factors.

The current status of the Bethe-Salpeter approach in Minkowski space was treated by V. Karmanov by employing the Nakanishi representation. In another keynote talk, P. Tandy considered applications of a computational scheme in hadron physics, which employs improved Bethe-Salpeter kernels within the set of Dyson-Schwinger equations. Predictions for the electromagnetic pion form factor were presented, which agree well with the existing data, obtained with a pion distribution amplitude which is significantly broader than the asymptotic one. Staying within the context of bound state systems, J.-F. Mathiot discussed the recently developed Taylor-Lagrange renormalization scheme applying it to the calculation of nonperturbative corrections to the mass of a fermion. The rich subject of AdS/QCD attracted the attention of several participants, among them, V. Lyubovitskij, A. Vega, and others, who treated the baryon structure within soft-wall AdS/QCD models. The pion-photon transition form factor was considered by J. de Melo and collaborators using a lightfront field theoretical approach to the constituent quark model and also by S. V. Mikhailov and collaborators within the framework of lightcone sum rules. Both approaches are in good agreement with the high- $Q^{2}$ Belle data but disagree with the BaBar data which indicate a growth with $Q^{2}$ of the scaled pion-photon transition form factor above $10 \mathrm{GeV}^{2}$. The last talk by G. Cvetic on the evaluation of spacelike QCD quantities in the IR regime in terms of a Landau-singularity free running coupling sparked intensive discussions and heated arguments among the participants. The reader can find more details about the mentioned papers and many other interesting contributions-all peer-reviewed—in this special issue.

Acknowledgments It is a pleasure to express my appreciation to my coorganizers and thank Martina Hacke and Peter Druck for their assistance in preparing and running the Conference. I am grateful to the Editorial Office (particularly Anjali Ratnajit) of Few Body Systems, which has done a superb job with the proceedings of this workshop. The financial support from the Gesellschaft der Freunde der Ruhr-Universität Bochum is gratefully acknowledged.

N. G. Stefanis

Chairman of the Organizing Committee and

Guest Editor of LC2013+

at Few Body Systems 